\title{
Alterações clínicolaboratoriais em pacientes com malária por Plasmodium vivax e deficiência de glicose-6-fosfato desidrogenase tratados com $0,50 \mathrm{mg} / \mathrm{kg} /$ dia de primaquina
}

\author{
Clinical and laboratorial alterations in Plasmodium vivax malaria patients and \\ glucose-6-phosphate dehydrogenase deficiency treated \\ with primaquine at $0.50 \mathrm{mg} / \mathrm{kg} /$ day
}

\author{
Mônica C.M. Silvaa ${ }^{1,2}$, Eliane B. Santos, Elenild G. Costa ${ }^{1}$, Manoel G.S. Filho', \\ João F. Guerreiro ${ }^{3}$ e Marinete M. Póvoa ${ }^{1}$
}

\begin{abstract}
RESUM0
0 efeito adverso da primaquina na dose de $0,50 \mathrm{mg} / \mathrm{kg} /$ dia foi investigado em onze pacientes com malária vivax ( três com deficiência de glicose-6-fosfato desidrogenase). Alterações clínicas e laboratoriais indicaram hemólise aguda apenas nos enzimopênicos, o que fez com que o tratamento fosse interrompido. Nossos resultados sugerem a necessidade do emprego de um teste de triagem para a deficiência de G6PD em áreas endêmicas de malária vivax a fim de se evitar complicações causadas pelo uso da primaquina.
\end{abstract}

Palavras-chaves: Deficiência de G6PD. Primaquina. Malária.

\begin{abstract}
The adverse effects of primaquine ( $0.50 \mathrm{mg} / \mathrm{kg} /$ day) were investigated in eleven patients with vivax malaria ( three patients with glucose-6-phosphate dehydrogenase deficiency). Clinical and laboratorial alterations indicated acute hemolysis in only the enzymopenic patients and treatment was interrupted. Our results suggest that screening for G6PD deficiency should be carried out in patients with vivax malaria infection in order to avoid complications due to primaquine.
\end{abstract}

Key-words: G6PD Deficiency. Primaquine. Malaria.

0 tratamento da malária causada por Plasmodium vivax é feito com a associação da cloroquina ( esquizonticida sanguíneo) e primaquina que é a droga de escolha para eliminar formas hipnozoíticas ${ }^{13}$. Diferentes estudos demonstram respostas variadas do parasita ao tratamento com primaquina e alguns autores sugerem 0 uso de doses acima da usual ${ }^{581216}$.

Devido ao seu caráter oxidante, a primaquina é capaz de induzir anemia hemolítica grave em pacientes com deficiência de glicose-6-fosfato desidrogenase(G6PD) ${ }^{7}$ mesmo na dose clássica de $0,25 \mathrm{mg} / \mathrm{kg} / \mathrm{dia}$, 0 que não ocorre em pacientes sem a enzimopatia ${ }^{25}$. A variante de G6PD mais freqüentemente associada a deficiência enzimática é a G6PD A; porém outras variantes tem sido descritas na literatura ${ }^{6}{ }^{9}$. Ainda, não está claro porque certos pacientes enzimopênicos não desenvolvem hemólise grave quando em contato com um agente oxidante ${ }^{2115}$. Porém, Kellermeyer et $\mathrm{al}^{11}$ sugeriram que a severidade não depende apenas de fatores intrínsecos dos eritrócitos, mas também de fatores extrínsecos como certas doenças sistêmicas e estados patológicos que podem potencializar a crise hemolítica. Segundo recomendações da WHO ${ }^{21}$, 0 tratamento da malária em pacientes enzimopênicos deve ser feito com a menor dose de primaquina $(0,25 \mathrm{mg} /$ $\mathrm{kg} /$ dia) ativa sobre 0 parasito, e nos casos onde a deficiência enzimática é severa, deverá ser evitada.

\footnotetext{
1. Instituto Evandro Chagas, Belém, PA. 2. Centro de Ensino Superior do Pará, Belém, PA. 3. Universidade Federal do Pará, Belém, PA. Endereço para correspondência: Dra. Mônica Cristina de Moraes Silva. Travessa Natal 3294, Cj. Bela Vista Val-de-Cães. 66125-010 Belém, PA. e-mail:monicamoraes@iec.pa.gov.br Recebido para publicação em 21/3/2003 Aceito em 20/4/2004
} 
Novos testes com dosagens modificadas de primaquina, que alteram 0 esquema clássico ( $0,25 \mathrm{mg} / \mathrm{kg} /$ dia durante 14 dias) para períodos menores de administração ( 5 a 7 dias) e dose diária dobrada têm sido utilizados para tratamento da malária por P. vivax em Belém ( Pará) ${ }^{1}$. Por isso, este estudo objetivou avaliar 0 efeito hemolítico da primaquina em pacientes maláricos com e sem a enzimopatia submetidos ao esquema de 0,50 mg/kg/dia.

\section{MATERIAL E MÉTODOS}

Pacientes. Concordaram em participar deste estudo, onze pacientes brasileiros, com idades entre 18 - 60 anos e peso acima de $60 \mathrm{~kg}$ e que não haviam utilizado medicamento de efeito oxidante 48 horas antes da coleta do sangue. Os pacientes chegaram ao Laboratório de Malária do Instituto Evandro Chagas/SVS com queixa de febre, calafrio, cefaléia, palidez cutânea e colúria. O diagnóstico de malária por P. vivax foi obtido por meio da técnica de gota espessa e coloração pelo Giemsa.

Triagem e caracterização da deficiência de G6PD. Após entrevista, feita por profissional médico, foram coletados $3 \mathrm{ml}$ de sangue venoso de cada participante para investigação da deficiência de G6PD utilizando teste de redução da metemoglobina ${ }^{4}$ e eletroforese em gel de agarose a $0,1 \%{ }^{10}$.

Tratamento. Os pacientes foram tratados e acompanhados por 6 meses pelo corpo médico do Ambulatório de Malária do Instituto Evandro Chagas, Belém, Pará. Como conduta terapêutica adotou-se a administração V.O. de cloroquina $10 \mathrm{mg} / \mathrm{kg}$ no $1^{0}$ dia, $7,5 \mathrm{mg} / \mathrm{kg}$ nos $2^{-}$e $3^{\circ}$ dias + primaquina $0,50 \mathrm{mg} / \mathrm{kg} / \mathrm{dia}$ do $1^{\circ}$ a 0 50 dias ( 3 pacientes) ou do $1^{0}$ ao 70 dias ( 8 pacientes) como proposto por Abdon et al ${ }^{1}$. Além da primaquina, nenhuma outra droga com efeito oxidante foi utilizada durante 0 tratamento.

Exames clínicolaboratoriais. Os efeitos adversos da primaquina foram avaliados através de acompanhamento clínico e laboratorial (testes hematológicos e bioquímicos do sangue). Os testes laboratoriais foram realizados até 3 dias após a interrupção ou término do tratamento. Investigaram-se niveis de hemácias (milhões $/ \mathrm{mm}^{3}$ ) , hemoglobina ( $\mathrm{g} / \mathrm{dL}$ ), hematócrito (\%) e volume corpuscular médio ( fL) utilizando-se equipamento de contagem eletrônica em aparelho CC-550 da CELM. Níveis de bilirrubina indireta foram obtidos por método colorimétrico.

Análise estatística. Os resultados dos parâmetros hematológicos e bioquímicos do sangue de pacientes enzimopênicos e não enzimopênicos foram utilizados como indicativos de processo hemolítico decorrente do uso da primaquina. Asignificância estatística foi testada pelo método ANOVA (análise de variância) através do programa BioEstat versão 2.0 ( Sociedade Civil Mamirauá/MCT-CNPq).

\section{RESULTAD0S}

Três pacientes apresentaram deficiência de G6PD caracterizada como variante $\mathrm{A}^{*}$, no estudo eletroforético.

Antes do tratamento, todos os pacientes (enzimopênicos e não enzimopênicos) relataram manifestações clínicas de malária (febre, calafrio, cefaléia, palidez cutânea, colúria) . A palidez cutânea e a colúria agravaram-se nos três enzimopênicos durante 0 decorrer do tratamento, e houve aparecimento de icterícia grave, hepatomegalia e esplenomegalia, tornando-se necessária a interrupção do mesmo. Dois enzimopênicos não relataram manifestações de hemólise durante uso de primaquina em infecções anteriores, cujo tratamento havia sido realizado com 0,50mg/kg/dia durante 7 dias. Todavia, um enzimopênico apresentou quadro hemolítico grave durante 0 primeiro contato com a droga.

A diminuição nos valores de hemoglobina e hematócrito e a presença de células vermelhas indiferenciadas ( anisocitose, eritroblastos ortocromáticos e policromatófilos) foram observados em todos os enzimopênicos.

Nenhuma alteração hematológica ou bioquímica foi encontrada entre os não enzimopênicos, porém dois enzimopênicos apresentaram níveis aumentados de bilirrubina indireta (Tabela 1).

Tabela 1 - Valores hematológicos ebioquímicos em pacientes enzimopênicos e não enzimopênicos tratados com 0,50mg/kg/dia de primaquina.

\begin{tabular}{lrrr}
\hline & $\begin{array}{c}\text { Pacientes não } \\
\text { enzimopênicos }\end{array}$ & $\begin{array}{c}\text { Pacientes } \\
\text { enzimopênicos }\end{array}$ & $P$ \\
\hline Hemoglobina & $12,73 \pm 1,38$ & $7,8 \pm 0,3$ & 0,0004 \\
Hemácia & $4,2 \pm 0,29$ & $2,62 \pm 0,22$ & 0,0001 \\
Hematócrito & $39,21 \pm 3,02$ & $24,63 \pm 1,01$ & 0,0001 \\
VCM* & $93,41 \pm 4,75$ & $94,13 \pm 8,39$ & 0,8484 \\
Bilirrubina indireta & $0,59 \pm 0,18$ & $1,03 \pm 0,32$ & 0,0168 \\
\hline
\end{tabular}

*VCM: Volume corpuscular médio

\section{DISCUSSÃ0}

0 Estado do Pará apresenta o maior número de casos de malária registrados na Região Amazônica, com a maioria dos casos causada por P. vivax. Vários estudos têm demonstrado freqüências polimórficas de variantes de G6PD causadoras de deficiência enzimática em populações desta região ( $2 \%$ segundo estudo de Santos et al ${ }^{18}, 6,1 \%$ Weimer et $\mathrm{al}^{20}$ e $8 \%$ Schneider et al ${ }^{19}$ ) e de outras cidades do Brasil ${ }^{17}$. A G6PD A ${ }^{\gamma}$ foi a única variante caracterizada neste estudo, porém a análise molecular permitirá a caracterização das mutações genéticas.

0 grau de manifestação da anemia hemolítica observada apenas nos enzimopênicos foi variável, o que corrobora os dados da WHO ${ }^{21}$. Em nosso estudo, demonstramos que o uso da primaquina agravou a hemólise causada pela própria malária e presentes nestes pacientes antes do tratamento.

Os valores de hemácias, hemoglobina e hematócrito foram evidentemente baixos quando comparados aos valores de referência para as populações. A diminuição destes parâmetros é indicativa de crise hemolítica em pacientes com deficiência de G6PD 23 .

A presença de células vermelhas indiferenciadas (anisocitose, eritroblastos ortocromáticos e policromatófilos) observada nos enzimopênicos, indica intensa eritropoiese necessária a reposição de hemácias perdidas por lise celular. 
Tais alterações não foram observadas nos não enzimopênicos. Isto demonstra que a hemólise decorrente da infecção malárica não foi suficiente para ativar com intensidade 0 sistema eritropoiético, sendo a hemólise produzida pela primaquina a principal responsável pela ativação celular. Tais alterações já foram observadas em indivíduos com deficiência de G6PD tratados com 0,50mg/kg/dia de primaquina, porém sem malária ${ }^{3}$.

0 nível normal de bilirrubina indireta em um enzimopênico pode ser evidência de que, provavelmente, o grau da deficiência seja fator importante para alteração deste parâmetro.

May eta ${ }^{14}$ estudando crianças com e sem deficiência de G6PD sem tratamento, não encontraram diferenças significativas quanto a anemia causada pela malária, 0 que nos faz concluir que as alterações clínicas e laboratoriais observadas em nossos pacientes foram causadas pela administração da primaquina.

Podemos concluir, que em nosso estudo as manifestações clínicas e os achados laboratoriais são sugestivos de processo hemolítico em pacientes com deficiência de G6PD tratados com primaquina.

Assim, sugerimos que a investigação da freqüência da deficiência de G6PD seja realizada em áreas endêmicas de malária vivax antes do emprego de esquemas terapêuticos com dosagens modificadas de primaquina, e que 0 teste de redução da metemoglobina ${ }^{4}$ por ter boa sensibilidade e baixo custo pode ser usado neste monitoramento. Todavia, caso necessário 0 emprego de primaquina nestes pacientes em dose acima da estabelecida pela $\mathrm{WHO}^{21}$, deve-se aplicar testes para quantificação do grau da deficiência enzimática, pois assim será possível determinar a inclusão e/ou quantidade da droga para tratamento.

\section{REFERÊNCIAS BIBLIOGRÁFICAS}

1. Abdon NP, Santos M, Pinto AYN, Calvosa VSP, Ventura A, Souza JM. Tratamento da malária vivax: esquema clássico x esquemas reduzidos. In: Resumos do XXXII Congresso da Sociedade Brasileira de Medicina Tropical, Brasília, p. 166, 1996.

2. Bangchang KN, Songsaeng W, Thanavibul A, Choroenlarp P, Karbwang J. Pharmacokinetics of primaquine in G6PD deficient and G6PD normal patients with vivax malaria. Transactions of the Royal Society of Tropical Medicine and Hygiene 88:220-222, 1994.

3. Beutler E, Dern RJ, Alving AS. The hemolytic effect of primaquine III: A study of primaquine-sensitive erythrocytes. The Journal of Laboratory and Clinical Medicine 2:177-184, 1954.
4. Brewer GJ, Tarlov AR, Alving AS. The methemoglobin reduction test for primaquine -type sensitivity of erythrocytes. Journal of the American Medical Association 180:386-388, 1962.

5. Bunnag D, Karbwang J, Thanavibul A, Chittamas S, Ratanapongse Y, Chalermrut K, Bangchang KN, Harinasuta T. High dose of primaquine in primaquine resistant vivax malaria. Transactions of the Royal Society of Tropical Medicine and Hygiene 88:218-219, 1994.

6. Calabrò V, Mason PJ, Filosa S, Civitelli D, Cittadella R, Tagarelli A, Martini G, Brancati C, Luzzatto L. Genetic heterogeneity of glucose-6-phosphate dehydrogenase deficiency revealed by single-strand conformation and sequence analysis. American Journal of Human Genetic 52:527-536, 1993.

7. Carson PE, Flanagan CL, Ickes CE, Alving AS. Enzymatic deficiency in primaquine-sensitive erythrocytes. Science 14:484-485, 1956.

8. Coatney GR, Getz ME. Primaquine and quinocide as curative agents against sporoozoite-induced Chesson strain vivax malaria: a preliminary report. Journal of National Malaria Society 9:285-289, 1962.

9. Corcoran CM, Calabrò V, Tamagnini G, Town M, Haidar B, Vulliamy TJ, Mason PJ, Luzzatto L. Molecular heterogeneity underlying the G6PD Mediterranean phenotype. Human Genetic 88:688-690, 1991.

10. Forbes J, Steytler GJ, Van Heerden R. Agarose gel electrophoresis of glucose-6phosphate dehydrogenase isoenzymes. Clinica Chimica Acta 199:279-282, 1991.

11. Kellermeyer RW, Tarlov AR, Brewer GJ, Carson PE, Alving AS. Hemolytic effect of therapeutic drugs: clinical considerations of the primaquine-type hemolysis. Journal of the American Medical Association 180:388-394, 1962.

12. Krostoski WA. Frequency of relapse and primaquine resistance in Southeast Asian vivax malaria. New England Journal of Medicine 303:587, 1980.

13. Manual de Terapêutica de Malária. Brasília, 1996.

14. May J, Falusi AG, Mockenhhaupt FP, Ademowo OG, Bienzle OU, Meyer CG. Impact of subpatent multi-species and multiclonal infections on anaemia in children from Nigeria. Transactions of the Royal Society of Tropical Medicine and Hygiene 94:399-343, 2000.

15. Menendez-Capote R, Diaz Perez L, Luzardo Suarez C. Hemolysis and primaquine treatment. Preliminary report. Revista Cubana de Medicina Tropical 49:136-138, 1997.

16. Olliaro P, Cattani J, Wirth D. Malaria, the submerged disease. Journal of the American Medical Association 3:230-233, 1996.

17. Saad STO, Salles TSI, Carvalho MHM, Costa FF. Molecular characterization of glucose-6-phosphate dehydrogenase deficiency in Brazil. Human Heredity 47:17-21, 1997.

18. Santos SEB, Guerreiro JF, Salzano FM, Weimer TA, Hutz MH, Franco MHLP. Mobility, blood genetic traits and race mixture in the Amazonian population of Oriximiná. Brazilian Journal of Genetic 4:745-759, 1987.

19. Schneider H, Guerreiro JF, Santos SEB, Weimer TA, Schneider MPC, Salzano, FM. Isolate breakdown in Amazonia - The blacks of the Trombetas river. Brazilian Journal of Genetic 3:565-574, 1987.

20. Weimer TA, Salzano FM, Westwood B, Beutler E. Molecular characterization of glucose-6-phosphate dehydrogenase variants from Brazil. Human Biology 65:41-47, 1993.

21. World Health Organization. Glucose-6-phosphate dehydrogenase deficiency. Bulletin of the World Health Organization 6:601-611, 1989. 